\title{
UNIFORM WORKLOAD DISTRIBUTION PROBLEMS
}

In this paper we review common studies from the years 1984-2015 of problems occurring in uniform scheduling of workload distributions. These problems were formulated first by Pesko in dealing with the practical problem of regular scheduling of service vehicles. Given the nonnegative, real matrix with daily records of vehicles as columns, we need to minimize some irregularity measure of row sums (workloads) by permuting matrix columns. The problem has various practical modifications, such as the weighted rows of matrix, workload uncertainty variance, graph approach of exchange of elements and generalized inverse formulations. Some of them are presented in the paper.

Keywords: Regular scheduling, matrix permutation, irregularity measure, NP-hard problem.

\section{Introduction}

In this paper we study problems which occur in uniform scheduling of workload distribution. The uniformity is expressed by an irregularity measure and the goal is to minimize the irregularity measure of parts of the schedule. This problem is motivated by the following job scheduling problem:

Let $n$ vehicles and $m \times n$ jobs be given. Every job has assigned to it the value ai,j which represents its quantity. These values form the $m \times n$ matrix $A$. We need to find the most regular m-days job schedule, which gives minimal difference between the sums of rows of the permuted version of the matrix $A$.

The problem is also called the Matrix Permutation Problem (MPP) which was first mentioned in [1]. Further investigation of MPP can be found in [2], where this problem was solved for garbage trucks.

The mathematical formulation of the problem is the following:

Let a nonnegative real $m \times n$ matrix $A=\left(a_{i j}\right)$ be given. Let $I=\{1,2, \ldots, m\}, J=\{1,2, \ldots, n\}$ be sets of row and column indices. For each column $j \in J$ of matrix $A$ we will use the notation $\pi_{j}$ for the permutations of elements in that column. Let $\pi=\left(\pi_{1}, \pi_{2}, \ldots, \pi_{n}\right)$ denote the vector of permutations of all columns of $A$ and let $A^{\pi}$ denote the permuted matrix. Let $P_{m n}$ be the set of all such permutation vectors. Let $S^{\pi}=\left(S_{1}^{\pi}, S_{2}^{\pi}, \ldots, S_{m}^{\pi}\right)$ denote the vector of row sums of permuted matrix $A^{\pi}$. Let $\mathrm{f}$ be a function called the irregularity measure. The following optimization problem will be called the uniform workload distribution problem (UWDP) $\min \left\{f\left(S^{\pi}\right) ; \pi \in P_{m n}\right\}$.
The irregularity measure $f: J^{m} \rightarrow\langle 0, \infty)$ is any Schur-convex function, where $J=(a, b)$ is an interval. More on irregularity measures and Schur-convex functions can be found in [3]. The most used irregularity measures are:

- $f_{s q r}^{\delta}\left(x_{1}, x_{2}, \ldots, x_{m}\right)=\left(x_{1}-\delta\right)^{2}+\left(x_{2}-\delta\right)^{2}+\ldots+\left(x_{m}-\delta\right)^{2}$

- $f_{a b s}^{\delta}\left(x_{1}, x_{2}, \ldots, x_{m}\right)=\left|x_{1}-\delta\right|+\left|x_{2}-\delta\right|+\ldots+\left|x_{m}-\delta\right|$

- $f_{\text {dif }}\left(x_{1}, x_{2}, \ldots, x_{m}\right)=\max \left(x_{1}, x_{2}, \ldots, x_{m}\right)-\min \left(x_{1}, x_{2}, \ldots, x_{m}\right)$

- $f_{\max }^{\delta}\left(x_{1}, x_{2}, \ldots, x_{m}\right)=\max \left(x_{1}, x_{2}, \ldots, x_{m}\right)-\delta$

- $f_{\min }^{\delta}\left(x_{1}, x_{2}, \ldots, x_{m}\right)=\delta-\min \left(x_{1}, x_{2}, \ldots, x_{m}\right)$

$\left(\right.$ where $\left.\delta=\min \left(x_{1}+x_{2}+\ldots+x_{m}\right) / m\right)$

\section{Computational complexity of UWDP}

In [4] it was proved that UWDP (MPP) is an NP-hard problem. In [2] it was shown that even a 2-row version of UWDP is NP-hard. The proof of this fact was established using transformation from the Set partition problem (definition of SPP can be found in [5]).

\footnotetext{
* 'Peter Czimmermann, ${ }^{1}$ Stefan Pesko, ${ }^{2}$ Jan Cerny

${ }^{1}$ Department of Mathematical Methods and Operations Research, Faculty of Management Science and Informatics, University of Zilina, Slovakia

${ }^{2}$ Department of Exact Methods, Faculty of Management, University of Economics, Prague, Czech Republic

E-mail: peter.czimmermann@fri.uniza.sk
} 
On the other hand, the two-column case is solvable in polynomial time. A simple polynomial algorithm can be found in [4]. It is enough to order the elements of the first column in a descending order and the elements of the second column in an ascending order. It is possible to show that we obtain the optimal solution for any irregularity measure mentioned above. The complexity of the algorithm based on this approach is $O(m \log m)$.

\section{Solutions of UWDP}

In this section we describe two heuristics for the solution of general UWDP. Both algorithms are based on the fact that the two-column case is solvable in polynomial time. We also consider the representation of UWDP by two models of mathematical programming.

\subsection{Decomposition method}

In the paper [2] the following heuristic was introduced:

Input: Matrix $A=A_{1}$ of the type $m \times n$.

For $i=1$ to $n-1$ do:

Create submatrix $B_{m \times 2}$ from the first pair of columns of $A_{i}$.

Solve the UWDP for matrix $B_{m \times 2}$. (The row-sum vector of the solution is denoted by $S^{\pi}$.)

Create matrix $A_{i+1}$ by replacing the first two columns of $A$ with column $S^{\pi}$.

Output: Solution for the matrix $A_{n-1}$.

Tests showed that this method is not very successful since the set of possible column permutations is very restricted.

\subsection{Stochastic decomposition method}

This method was introduced and studied in [6 and 7]. Let $J_{1}$ be the nonempty proper subset of column indices $\mathrm{J}$ and $J_{2}=J-J_{1}$. The set $\left[J_{1}, J_{2}\right]$ will be called the admissible decomposition of the columns of matrix $A$.

S1: Let $\pi=\left(\pi_{1}, \pi_{2}, \ldots, \pi_{n}\right)$ be arbitrary permutations of index set $I$.

S2: Choose randomly $\left[J_{1}, J_{2}\right\}$ - admissible decomposition of the columns of $A$.

S3: Solve the two-column UWDP with aggregated matrix $B=\left(b_{i j}\right)_{(m \times 2)}$

where $b_{i 1}=\sum_{j \in J_{1}} a_{\pi_{j}(i), j}, b_{i 2}=\sum_{j \in J_{2}} a_{\pi_{j}(i), j}, i \in I$
We get optimal column permutations $\varphi_{1}, \varphi_{2}$, satisfying conditions

$b_{\varphi_{1}(1), 1} \leq b_{\varphi_{1}(2), 1} \leq \cdots \leq b_{\varphi_{1}(m), 1}$,

$b_{\varphi_{1}(1), 2} \leq b_{\varphi_{2}(2), 2} \leq \cdots \leq b_{\varphi_{2}(m), 2}$.

S4: Update $\pi$ by applying permutation $\varphi_{1}\left(\operatorname{resp} . \varphi_{2}\right)$ to $\pi_{j}$ for $j \in J_{1}\left(\right.$ or $\left.j \in J_{2}\right)$.

S5: If no stopping criterion is satisfied GOTO S2, else END, $A^{\pi}=\left(a_{\pi_{j}(i), j}\right)$.

Tests on real data give promising results. The authors Š. Peško and M. Kaukič state conjecture that this algorithm can also be (with a suitable iteration count and sufficient number of restarts) the exact algorithm (at least for irregularity measure $f_{d i j}$ ).

\subsection{Model of linear programming}

In the mentioned work [7] the following model of mixed, integer, linear programming (MILP) was introduced. Objective function is $f_{d i f}$.

$$
\begin{aligned}
& \begin{array}{ll}
\text { s.t. } \sum_{k \in I}^{z_{U}-z_{L} \rightarrow \min } x_{i j k}=1 & \forall(i, j) \in I \times J, \\
\sum_{i \in I} x_{i j k}=1 & \forall(k, j) \in I \times J,
\end{array} \\
& z_{i}=\sum_{(j, k) \in J \times I} a_{k j} x_{i j k} \quad \forall i \in I, \\
& x_{i j k} \in\{0,1\} \quad \forall(i, j, k) \in I \times J \times I \\
& z_{L} \leq z_{i} \leq z_{U} \quad \forall i \in I, \\
& S_{L} \leq z_{L} \leq z_{U} \leq S_{U} \text {. }
\end{aligned}
$$

The value of $x_{i j k}$ is equal to one if $\pi_{j}(i)=k$ and otherwise zero. The real variables $z_{i} i \in I$ are the i-th row sums of permuted matrix $A^{\pi}$ and the variables $z_{L}, z_{U}$ are variables for lower and upper bounds $S_{L}, S_{U}$ of row sums.

\subsection{Model of quadratic programming}

In [7] the model of mixed, integer, quadratic programming (MIQP) was introduced. Objective function is $f_{s q r}^{\delta}$.

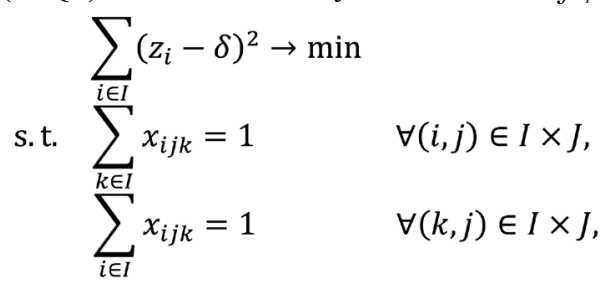




$$
\begin{array}{ll}
z_{i}=\sum_{(j, k) \in J \times I} a_{k j} x_{i j k} & \forall i \in I, \\
x_{i j k} \in\{0,1\} & \forall(i, j, k) \in I \times J \times I \\
S_{L} \leq z_{i} \leq S_{U} & \forall i \in I .
\end{array}
$$

Variables $x_{i j k}$ and $z_{i}$ mean the same as in the MILP model. Tests show that the MILP model is more effective than the MIQP model, but the mathematical programming solvers (even of such quality as Gurobi) have sometimes difficulties with the solving of not very large UWDP instances in reasonable time (since the model has large number of bivalent variables).

\section{Restricted sets of permutations}

In [8] the generalization of UWDP was suggested in which the set of permutations is restricted. The permitted permutations are represented by graphs. Paper [9] deals with conditions which allow to represent the set of permitted permutations by graphs The two-column case of this generalization is studied in [10]. It was shown that this problem can be solved as the most regular perfect matching in a graph. In the mentioned paper [10], there are introduced exact polynomial algorithms for finding the most regular perfect matching in graphs for irregularity measures $f_{\text {dif }}, f_{\max }^{\delta}$ and $f_{\min }^{\delta}$. The existence of exact polynomial algorithms for measures $f_{s q r}^{\delta}, f_{a b s}^{\delta}$ was an open problem.

We will show that there is also polynomial algorithm for irregularity measure $f_{s q r}^{\delta}$, but the weights of edges must satisfy a special property (which is fulfilled by graphs that arise from the mentioned generalization of UWDP). Let an edge-weighted graph $G=(V, E, w)$ be given. Let $V=\left\{v_{1}, v_{2}, \ldots, v_{2 m}\right]$. Let $w: E \rightarrow R_{0}^{+}$be a function that represents the weights of edges. If there exists a mapping $x: V \rightarrow R_{0}^{+}$such that

$\forall e \in E e=\left\{v_{i}, v_{j}\right\} w(e)=x\left(v_{1}\right)+x\left(v_{j}\right)$

we say that $\mathrm{w}$ is induced by $x$. There exist mappings $w$ that cannot be induced by any $x$. For example, we can consider complete graph on 4 vertices $v_{1}, v_{2}, v_{3}, v_{4}$ with edge weights

\begin{tabular}{|c|c|c|c|c|c|c|}
\hline edge & $\left\{v_{1}, v_{2}\right\}$ & $\left\{v_{1}, v_{3}\right\}$ & $\left\{v_{1}, v_{4}\right\}$ & $\left\{v_{2}, v_{3}\right\}$ & $\left\{v_{3}, v_{4}\right\}$ & $\left\{v_{3}, v_{4}\right\}$ \\
\hline weight & 2 & 2 & 2 & 4 & 2 & 4 \\
\hline
\end{tabular}

This leads to the unsolvable system of linear equations:

$$
\begin{aligned}
& x_{1}+x_{2}=2 \\
& x_{1}+x_{3}=2 \\
& x_{1}+x_{4}=2 \\
& x_{2}+x_{3}=4 \\
& x_{2}+x_{4}=2 \\
& x_{3}+x_{4}=4
\end{aligned}
$$

where $x_{1}, x_{2}, x_{3}, x_{4}$ are possible values of vertices. Hence $\mathrm{w}$ cannot be induced by any $x$.

Let $G=(V, E, w, x)$ be a graph. Let $V=\left\{v_{1}, v_{2}, \ldots, v_{2 m}\right\}$ and $\mathrm{w}$ be a mapping on edge set induced by mapping $\mathrm{x}$ on vertex set. Then the problem of finding the most regular perfect matching is the problem to find such perfect matching in $\mathrm{G}$, for which the function $f_{s q r}^{\delta}$ is minimal. The weight of edge $e_{i}$ will be denoted by $w_{i}$. If the perfect matching contains the edges $e_{1}, e_{2}, \ldots, e_{m}$, then we have

$f_{s q r}^{\delta}\left(w_{1}, w_{2}, \ldots, w_{m}\right)=\sum_{i=1}^{m}\left(w_{i}-\delta\right)^{2}$

where $\delta=\left(w_{1}+w_{2}+\cdots+w_{m}\right) / m$. Since the matching is perfect (it contains all vertices of $\mathrm{G}$ ) and the edge weights are induced by vertex values, we obtain:

$\sum_{i=1}^{m} w_{i}=\sum_{j=1}^{2 m} x\left(v_{j}\right)=m \delta$

The last sum is taken over all vertices of $G$ and hence it is a constant. The consequence is that $\delta$ is a constant for given graph $G$ and mapping $x$. Then

$\sum_{i=1}^{m}\left(w_{i}-\delta\right)^{2}=\sum_{i=1}^{m}\left(w_{i}^{2}-2 \delta w_{i}+\delta^{2}\right)=$

$\sum_{i=1}^{m} w_{i}^{2}+m \delta^{2}-2 \delta \sum_{i=1}^{m} w_{i}=\sum_{i=1}^{m} w_{i}^{2}+m \delta^{2}-2 m \delta^{2}=$

$\sum_{i=1}^{m} w_{i}^{2}+m \delta^{2}$

It means that the problem to find minimum of the function $f_{s q r}^{\delta}$ is in our case equivalent to the problem to find the minimum of the function $f_{s q r}$. Hence the problem of finding the most regular perfect matching in a graph $G=(V, E, w, x)$ is equivalent to the problem of finding minimal perfect matching in graph $G=\left(V, E, w^{2}\right)$ and this problem is solvable in polynomial time [11].

\section{Conclusions and further research}

There are several approaches how to represent uncertainty. In [12] the UWDP in interval arithmetic was introduced. It means that elements of matrix A are not exact values, but we have intervals, to which these values belong. It was proved that this problem is NP-complete and the two-column case is solvable in polynomial time.

The most common approach in systems with uncertainty is the fuzzy arithmetic. Our future plan is to define the UWDP in fuzzy arithmetic. We suppose that the complexity results will not change but it remains an open problem.

The weighted version of the problem was studied in [13]. It is called the weighted uniform workload distribution problem 
(WUWDP). Let $S^{\pi}=\left(s_{1}^{\pi}, s_{2}^{\pi}, \ldots, S_{m}^{\pi}\right)$ denote the vector of row sums. Let $w_{1}, w_{2}, \ldots, w_{m}$ be weights of rows. In this version of the problem we require that the values $w_{1}, s_{1}^{\pi}, w_{2} s_{2}^{\pi}, \ldots, w_{m} s_{m}^{\pi}$ are as uniform as possible. It is easy to consider that the UWDP is a special case of weighted version, where $w_{1}=w_{2}=\ldots=w_{m}=1$.

At the end, we can also mention some works which deal with similar problems to the UWDP. For example: the column permutation algorithm of a special case of the UDWP is studied in papers [14] and [15], the uniform k-partition problems are classified and examined in [16 and 17].

\section{Acknowledgment}

The research of P. Czimmermann was supported by the Scientific Grant Agency of the Ministry of Education of the Slovak Republic, the Slovak Academy of Sciences under project VEGA 1/0518/15 "Resilient rescue systems with uncertain accessibility of service", and the research of Š. Peško was supported by the Slovak Research and Development Agency under projects APVV-0760-11 "Designing of fair service systems on transportation networks" and APVV-14-0658 "Optimization of urban and regional public personal transport".

\section{References}

[1] COFFMAN, E. G., YANNAKAKIS, M.: Permuting Elements within Columns of a Matrix in order to Minimize Maximum Row Sum, Mathematics of Operations Research, 9(3):384390, 1984.

[2] CERNY, J., VASEK, K., PESKO, S., PALUCH, S., ENGELTHALLER, D.: Transport Scheduling and its Optimization (in Slovak), Research report III-8-9/03, Research Institute of Transport, 1986.

[3] CERNY, J., KLUVANEK, P.: Principles of Mathematical Theory of Transport (in Slovak), VEDA, Bratislava, 1991.

[4] TEGZE, M., VLACH, M.: On the Matrix Permutation Problem, Zeitschrift fur Operations- Research, 30(3), 1986.

[5] GAREY, M. R., JOHNSON, D. S.: Computers and Intractability, A Guide to the Theory of NP-completeness, Freeman: San Francisco, 1979.

[6] PESKO, S., HAJTMANEK, R.: Matrix Permutation Problem for Fair Workload Distribution, Mathematical Methods in Economics, $32^{\text {nd }}$ international conference, Olomouc, 2014.

[7] PESKO, S., KAUKIC, M.: Stochastic Algorithm for Uniform Workload Distribution Problem, Proc. of $13^{\text {th }}$ Intern. Conference Informatics, IEEE, Poprad, 2015.

[8] CZIMMERMANN, P., PESKO, S.: The Regular Permutation Scheduling on Graph, J. of Information, Control and Management Systems, vol. 1, No. 1, 2003.

[9] CZIMMERMANN, P.: A Note on using Graphs in Regular Scheduling Problems, Communications - Scientific Letters of the University of Zilina, vol. 5, No. 4, 2003.

[10] CZIMMERMANN, P.: The Most Regular Perfect Matchings in Graph, Studies of the University of Zilina, Mathematical Series, vol. 16, No. 1, 2003.

[11] EVEN, S., KARIV, O.: An Algorithm for Maximum Matching in General Graphs, Proc. of $16^{\text {th }}$ Annual Symp. on Foundations of Computer Science IEEE, New York, 1975.

[12] PESKO, S.: The Matrix Permutation Problem in Interval Arithmetic, J. of Information, Control and Management Systems, vol. 4, No.1, 2006.

[13] PESKO, S., CERNY, J.: Uniform Splitting in Managerial Decision Making, Economics and Management, 9(4), 2006.

[14] PUCCETTI, G., RUSCHENDORF, L.: Computation of Sharp Bounds on the Distribution of a Function of Dependent Risk, J. of Computational and Applied Mathematics, 236(7), 2012.

[15] BOUDT, K., VANDUFFEL, S., VERBEKEN, K.: Block Rearranging Elements within Matrix Columns to Minimize the Variability of Row Sums, http://ssrn.com/abstract=2634669, 2015.

[16] DELl'OlMO, P., HANSEN, P., PALlOTTINO, S., STORCHI, G.: On Uniform k-partition Problems, Discrete Applied Mathematics, 150(1-3), 2005.

[17] PESKO, S.: Minimal Total Area Convex Set Partitioning Problem, Communications - Scientific Letters of the University of Zilina, vol. 11, No. 3, 2009. 